\title{
Avaliação da Influência de Fatores Sociais e Econômicos no Uso da Metodologia PBL + m-learning para o Ensino da Matemática
}

\author{
Tarcisio Tavares Sanches, UFPA, tarcisiosanches1007@gmail.com \\ Miguel Ângelo Rodrigues Mocbel, UFPA, angelomocbel@gmail.com \\ Vicente Caldas de Castro, UFPA, vicente_soad@yahoo.com.br \\ Carlos Augusto Machado dos Santos, UFPA, carlos25.asm@gmail.com \\ Leonardo Gonçalves Martins, UFPA, leonardo20.gm@gmail.com \\ Waldma Maíra Menezes de Oliveira, UFPA,waldmamaira@hotmail.com \\ Álvaro Júnior Melo e Silva, UFPA, alvarojunior.4@hotmail.com \\ Fabricio de Souza Farias, UFPA, fabriciosf@ufpa.br
}

\begin{abstract}
Resumo. Embora o método de ensino tradicional seja predominantemente adotado nas escolas, os índices de avaliação estudantis apresentam dados alarmantes que ilustram a necessidade da busca por novas metodologias permanentes para o aprendizado. Diversos pesquisadores apresentam trabalhos que apontam o aprendizado baseado em problemas (PBL) e o aprendizado baseado no uso de plataformas móveis (m-learning) como caminhos para melhoria do desempenho dos estudantes. Este artigo analisa de modo tridimensional o uso do PBL e m-learning, considerando fatores sociais $e$ econômicos no contexto da disciplina de matemática. A partir deste estudo, é possível inferir que fatores sociais e econômicos influenciam no sucesso da metodologia proposta, assim como, o impacto da metodologia no desempenho dos estudantes.
\end{abstract}

Palavras-Chave: aprendizado, PBL, m-learning, matemática.

\section{Evaluation of the Influence of Social and Economic Factors in the Use of the PBL + M-learning Methodology for Teaching Mathematics}

\begin{abstract}
Although the traditional teaching method is predominantly adopted in schools, the student evaluation indices present alarming results that illustrate the need of new investigations about methodologies for learning. Several researchers present papers that point to problem-based learning (PBL) and learning based on the use of mobile platforms (m-learning) as ways to improve students' performance. This paper analyzes in a threedimensional way the use of PBL and m-learning, considering social and economic factors in the context of the mathematics discipline. From this study, it is possible to infer that social and economic factors cause influence in the success of the proposed methodology, as well as the impact of the methodology on students' performance.
\end{abstract}

Keywords: learning, PBL, mobile learning, math.

\section{Introdução}

Proporcionar equilíbrio e condições favoráveis para o alcance do aprendizado pleno por parte dos estudantes e para a melhoria da qualidade do ensino oferecido pelos professores, são desafios constantes para todos os agentes envolvidos no processo de ensino-aprendizagem, isto é, professores, alunos, pais de alunos e gestores. Historicamente, os agentes buscam o equilíbrio e condições favoráveis fazendo uso do modelo tradicional de ensino, o qual foca no compartilhamento verbal de conteúdos para os alunos que atuam de forma passiva durante o processo (Mizukami, 1986) (Cyrino e Toralles-Pereira, 2004). 
Este modelo é predominantemente baseado no uso clássico do quadro branco e explanação do professor. Além disso, pode ser considerado ferramenta excludente, pois privilegia determinado perfil de alunos que se adequam à metodologia e excluí os que não se adaptam ao modelo implementado (Jacomini, 2009); apresentando assim poucas opções e funcionando como um limitador do desenvolvimento de um conjunto de alunos que possuem diferentes interesses e repertórios comportamentais. A carência de diversificação metodológica pode refletir nos índices de desempenho escolar (Felício e Fernandes, 2005), como pode ser observado no relatório público do Exame Nacional do Ensino Médio de 2018 (Relatório ENEM, 2018). Tal fato abre precedente para se cogitar a necessidade da adição de novas metodologias de ensino no dia a dia escolar. Nesse sentido, podemos citar o aprendizado baseado em problema e o aprendizado baseado no uso de plataformas móveis.

$\mathrm{O}$ aprendizado baseado em problemas ou problem-based learning (PBL) é um recurso educacional, sendo uma ferramenta didática inovadora, que vem obtendo muitos seguidores. Este foi proposto em 1920 na escola de direito da Universidade de Harvard, nos Estados Unidos da América (EUA), em seguida foi investigado e melhorado pela escola de medicina na Universidade de McMaster, no Canadá, no final de 1960 (HmeloSilver, 2004). O objetivo do PBL é construir conhecimento a partir da interação dos alunos, considerando a soma do conhecimento de cada um, tornando assim possível chegar à construção do saber (Jung, 2015), o que corrobora com as perspectivas de uma educação dialógica, libertadora e baseada na solução de problemáticas. Segundo Freire (1994) a educação é um ato de conhecimento em que ambos, educador e educando atuam como sujeitos cognoscentes, mediatizados pelo mundo.

Sales (2016) destaca que o PBL segue as últimas diretrizes e parâmetros curriculares da educação básica, reduzindo a diferença entre o aprendizado da teoria e a prática, assim como, transformando o professor como agente que estimula a participação dos alunos. Com esse método, o foco do ensino passa a ser o aluno, que deixa de exercer o papel apenas de receptor passivo das informações transmitidas por seus professores.

Já o aprendizado baseado em plataformas móveis ou mobile-learning ( $\mathrm{m}$ learning) é uma estratégia de aprendizagem que propõe a democratização do acesso a diversos conteúdos educativos. É um conceito amplo que foca na disponibilização do acesso à informação de forma independente a qualquer instituição ou ambiente físico. $\mathrm{O}$ m-learning se tornou uma realidade em virtude do avanço das tecnologias móveis, isto é, o ramo da educação passou a se estender aos dispositivos móveis, como smartphones e tablets. Esta liberdade permite aos usuários o acesso a conteúdos provenientes de diferentes fontes, por exemplo, aplicativos ou vídeos educacionais desenvolvidos para plataformas móveis. Atualmente, a produção de novos conteúdos e materiais didáticos para m-learning cresce exponencialmente, principalmente para a plataforma Android, onde aplicativos para educação lideram entre as categorias de softwares desenvolvidos (Top 10 Google Play Categories, 2019).

Embora existam diversos trabalhos que apresentem propostas metodológicas baseadas em PBL e m-learning, suas análises limitam-se predominantemente em avaliar de modo separado o impacto de cada uma das estratégias em relação ao método tradicional de ensino, assim como, não levam em consideração fatores sociais e econômicos, tais como infraestrutura e condições dos lares, acesso a recursos tecnológicos ou localização residencial (área rural ou urbana) (Ribeiro, 2008) (Andrade et. al., 2005) (Franciscato e Medina, 2008). A partir deste contexto, este trabalho objetiva realizar uma análise do impacto da utilização conjunta das metodologias baseadas em PBL e m-learning como estratégia de aprendizagem na disciplina de matemática, assim como, visa analisar o impacto de fatores sociais e econômicos em relação ao alcance pleno 
do ensino e aprendizagem nessa área do conhecimento. Para isso, foi realizada uma análise tridimensional que leva em consideração os seguintes itens de avaliação: método de ensino e aprendizagem adotado (tradicional ou baseado em PBL $+m$-learning), fator social (composição familiar) e fator econômico (infraestrutura de moradia e localidade).

O restante deste trabalho está organizado da seguinte forma: na Seção II é apresentado uma revisão bibliográfica sobre PBL e m-learning, na Seção III é apresentado a metodologia de aplicação das técnicas, na Seção IV são apresentados os resultados obtidos, por fim, são apresentadas as considerações finais do trabalho.

\section{Revisão Bibliográfica}

Na literatura, PBL e m-learning são apresentados e tratados de formas distintas, o que mostra a necessidade da apresentação de uma abordagem que considere os pontos positivos de ambas as metodologias de forma conjunta. Nesta seção, apresentaremos diversos autores que tratam sobre ambas as metodologias de forma separada. Para a realização do levantamento bibliográfico foi utilizado o repositório de artigos do Google Acadêmico, sendo utilizadas na busca as seguintes palavras-chave: "PBL", "m-learning", "PBL+m-learning", "Problem-Based Learning" e "Mobile Learning".

\subsection{Problem-Basead Learning}

O PBL é uma estratégia de ensino focada em autoaprendizagem, a qual torna o aluno um agente participativo na busca do conhecimento (Andrade et. al., 2005). Para ressaltar a concepção desta estratégia de ensino, seus autores apresentaram a aplicação da metodologia PBL na disciplina de biologia, com alunos do $2^{\circ}$ ano do ensino médio. $\mathrm{O}$ experimento foi dividido em duas etapas. Na primeira etapa, o aluno poderia avaliar os colegas de turma, enquanto na segunda etapa o aluno deveria testar suas habilidades frente aos problemas apresentados, avaliando o conteúdo que foi repassado no grupo de estudo no decorrer da aplicação do PBL.

Ribeiro (2008) destaca que a metodologia PBL não é uma estratégia pronta a ser trabalhada, mas sim um caminho a ser formado de acordo com as dificuldades encontradas. Desta forma, ao término da aplicação do PBL, os estudantes devem descrever suas impressões em relação à aplicação da nova metodologia. Na mesma lógica, Queiroz (2012) complementa destacando que o aluno passa a ser agente ativo no processo de ensino e aprendizagem.

Em consonância com os conceitos de Ribeiro (2008) e Queiroz (2012), Mezzari (2011) traz uma abordagem sobre a introdução do PBL na disciplina de Parasitologia e Micologia do curso de Medicina da Universidade Federal de Ciências da Saúde de Porto Alegre (UFCSPA), onde esse curso apresentava parte de sua metodologia na modalidade à distância, e era nesse intervalo que o PBL se fazia presente. Esse trabalho se deu de março a julho de 2009, contendo um total de 44 alunos/participantes. As avaliações foram realizadas com os alunos e tinham por objetivo mostrar que o método que estava sendo implantado para a turma era realmente eficaz. Para validar a investigação, foram realizadas diversas avaliações com os alunos. Durante as avaliações, os alunos foram separados em dois grupos, onde um foi submetido à aplicação do PBL (experimental) e o outro grupo não foi (grupo controle). O intuito de separar os grupos foi de comparar a nota final de ambos e mostrar o nível desempenho de cada um. Por fim, os resultados dessa pesquisa demonstram que os participantes que fizeram parte da aplicação do PBL, tiveram um rendimento bastante significativo. Os participantes também relataram sobre a importância do método, por ser de fácil acesso e sem custo para os usuários. 
Leite (2005) fez uma abordagem sobre a opinião dos alunos em relação à metodologia PBL. Esta pesquisa foi desenvolvida na Universidade do Minho e teve como participantes alunos da turma de licenciatura em física e química. $\mathrm{O}$ resultado dessa pesquisa também mostrou um alto índice de motivação e envolvimento por parte dos participantes que usaram PBL. O autor relatou também um aumento na autonomia dos alunos.

\section{$2.2 \quad$ M-Learning}

Pesquisas apresentadas pela literatura destacam a importância do uso do $\mathrm{m}$ learning no cenário educacional. Este recurso tem como principal característica a mobilidade, isto é, são softwares instalados em dispositivos que podem ser utilizados e transportados para todos os lugares.

De Oliveira e Medina (2007) frisa a importância dos dispositivos digitais na assistência aos professores em suas aulas proporcionando momentos com maior interatividade e dinamismo, favorecendo uma maior compreensão dos conteúdos que estão sendo trabalhados em sala, podendo tornar o ensino mais eficaz. Para reforçar essa informação, os pesquisadores destacam que o ensino com o auxílio de ferramentas móveis vem passando por uma crescente evolução em função, por exemplo, do avanço tecnológico.

Franciscato e Medina (2008) destaca em seu trabalho que embora o m-learning seja uma estratégia contemporânea, isso não significa que ele não consiga se enquadrar nos modelos educacionais. Além disso, o autor ressalta ainda que essa ferramenta não retém o seu usuário em apenas um local, bem diferente do modelo utilizado no método tradicional de ensino. Por fim, Batista e Barcelos (2017) apresentam uma análise sobre o uso do aparelho celular na sala de aula e realizam uma discussão sobre o seu papel como objeto de auxilio ou desconcentração. Para legitimar a pesquisa, um experimento foi realizado com estudantes do curso de licenciatura em matemática de uma universidade federal. Com base no estudo, os autores declaram que o uso do dispositivo eletrônico ainda é um assunto delicado a ser abordado e que precisa de ampla investigação, seja dentro ou fora do perímetro escolar, mesmo achando importante unir ferramentas tecnológicas como auxilio para os alunos nas aulas.

\section{Metodologia de Aplicação}

A aplicação da proposta PBL+m-learning se deu a partir de etapas, que envolveram o uso do software Exatas (Mocbel e Farias, 2018) no contexto educacional e o levantamento de dados dos alunos participantes. O aplicativo Exatas consiste em uma solução para plataformas móveis que aborda o estudo de disciplinas de matemática, física e química em um único ambiente. O Exatas é uma ferramenta desenvolvida para propiciar o melhor aprendizado baseado em problemas, isto é, apresenta aos seus usuários diversos conteúdos dispostos na forma de teoria, exercícios interativos de múltipla escolha, resolução de exercícios na forma de vídeos, e apresentação de conteúdos teóricos na forma de vídeos.

Com o aplicativo publicado, deu-se início à seleção e preparação do material disponível no aplicativo, afim de ser utilizado nas etapas de testes, sendo o assunto escolhido: Funções. O próximo passo consistiu na produção de novos vídeos para o assunto selecionado. Em seguida, a coleta de dados se deu por meio de questionários, uma vez que "possibilita investigações mais profundas e precisas [...]" (Lakatos e Marconi, 2003, p. 204), sendo eles, questionários de testes e coleta de informações 
socioeconômicas, na qual foram elaborados com base na literatura (FADESP, 2016). Os questionários de coleta de informações visam categorizar os alunos por questões socioeconômicas, como infraestrutura e localização de moradia, acesso a recursos tecnológicos, participação em programas sociais públicos, entre outras. Com isso, foi feita a seleção da turma convidada para os testes. Para este estudo, foi obtida a participação de 26 alunos voluntários.

$\mathrm{Na}$ realização dos testes, os participantes foram convidados a preencher o questionário socioeconômico, onde responderam perguntas sobre moradia (condições residenciais, bairro e saneamento), grau de escolaridade dos pais e participação em programas sociais fomentados pelo Governo. Em seguida, os alunos passaram a responder uma prova contendo sete questões de matemática sobre assuntos de funções, denominada de pré-teste. O principal objetivo do pré-teste foi verificar o conhecimento prévio dos alunos em relação ao assunto abordado.

Ao final do pré-teste, a turma foi dividida aleatoriamente em dois grupos iguais, no caso, 13 alunos. Nesta etapa, foi determinado um tempo de trinta minutos para cada grupo participar das aulas. O primeiro grupo participou de uma aula tradicional que tratou sobre os assuntos abordados no pré-teste, enquanto que o segundo grupo foi convidado a utilizar como estratégia de aprendizagem o PBL + m-learning. Para esta abordagem, foi indicado aos alunos, o uso do software Exatas como ferramenta de auxilio, que já continha os vídeos produzidos e materiais atualizados sobre o assunto abordado no pré-teste. Os alunos foram orientados a acessar o conteúdo e também foram acompanhados por tutores especializados na área de educação e ensino.

Ao final das aulas, os alunos foram convidados a voltar para a sala de aula principal, onde foi apresentado o formulário de avaliação. Neste, os alunos do segundo grupo avaliaram seu nível de aceitação da proposta PBL + m-learning em relação ao método tradicional. Após isso, todos os alunos foram submetidos a mais uma prova, denominada de pós-teste, sendo esta prova elaborada conforme a do pré-teste, isto é, sete questões sobre o mesmo assunto abordado.

Após a realização dos testes, os resultados dos questionários foram coletados e agrupados a fim de se analisar as relações entre: o desempenho dos alunos nos testes, o método de ensino utilizado e os fatores sociais e econômicos.

\section{Resultados}

Esta seção apresenta os resultados obtidos durante o experimento. Vale ressaltar que a aplicação dos testes realizados vai além de somente identificar se o método PBL + m-learning é mais eficiente em relação ao tradicional, pois a aplicação desta metodologia também visa verificar se fatores sociais e econômicos influenciam na eficiência dos métodos tradicionais e PBL + m-learning.

O Quadro 1 apresenta a distribuição social e econômica de acordo com as respostas obtidas durante as entrevistas. É possível observar a estrutura de moradia e nível de escolaridade dos pais assim apresentando dados distribuídos em diferentes perfis e realidades de cada aluno, o que permite diferentes análises que serão apresentadas nesta seção.

Primeiro, analisou-se o índice de aceitação do método PBL + m-learning quando comparado ao método tradicional, os resultados demonstram que o novo método teve aceitação bastante satisfatória em relação às perguntas realizadas, como: se aprovam a metodologia proposta em relação ao método tradicional; se o mesmo supre as expectativas; se indicariam para um amigo; se pretendem continuar usando o método apresentado; e se consideram o método melhor do que o método tradicional, sendo as 
respectivas porcentagens de aprovação obtidas para cada uma das questões apresentadas aos entrevistados: 95\%, 93\%, 90\%, 93\% e 98\%, isto é, obtendo média superior a $93 \%$.

Em relação ao desempenho dos alunos após as etapas de pré-teste e pós-teste, os resultados demonstram significativo aumento no desempenho por parte dos alunos após a aplicação do PBL + m-learning e aula tradicional, isto é, o índice de acerto na etapa de pós-teste foi superior à etapa de pré-teste. Na Figura 1, apresentamos o desempenho dos alunos no pré e pós-teste, quando avaliados pelo método tradicional e pelo método PBL+ m-learning, estes dados fazem referência aos artigos estudados nesta pesquisa nos quais a literatura aponta que a metodologia PBL obtinha melhores resultados quando avaliados frente ao método tradicional de ensino.

\section{Quadro 1 - Índice social e econômico dos participantes.}

\begin{tabular}{|c|c|c|c|c|c|}
\hline \multicolumn{6}{|c|}{ Dados Socioeconômicos } \\
\hline \multicolumn{2}{|c|}{ Situação de Moradia } & \multicolumn{2}{|c|}{ Dados dos Participantes } & \multicolumn{2}{|c|}{ Nível de Escolaridade dos Pais } \\
\hline Bairros Centro & 6 & Número de Participantes & 26 & Ensino Superior completo & 7 \\
\hline Bairros Periféricos & 20 & $\begin{array}{l}\text { Média de Idade dos } \\
\text { Participantes }\end{array}$ & 20 & $\begin{array}{l}\text { Ensino Superior } \\
\text { Incompleto }\end{array}$ & 0 \\
\hline $\begin{array}{l}\text { Possuem Imóvel } \\
\text { Próprio }\end{array}$ & 21 & $\begin{array}{l}\text { Recebem Benefícios do } \\
\text { Governo }\end{array}$ & 16 & Ensino Médio completo & 13 \\
\hline $\begin{array}{l}\text { Possuem Rua } \\
\text { Asfaltada }\end{array}$ & 15 & $\begin{array}{l}\text { Consideram seu Bairro } \\
\text { Violento }\end{array}$ & 11 & Ensino Médio incompleto & 9 \\
\hline $\begin{array}{l}\text { Possuem Rede de } \\
\text { Esgoto }\end{array}$ & 7 & Possuem Acesso à Internet & 16 & $\begin{array}{l}\text { Ensino Fundamental } \\
\text { completo }\end{array}$ & 5 \\
\hline Possuem Luz Elétrica & 26 & $\begin{array}{l}\text { Possui Acesso a Posto de } \\
\text { Saúde no bairro }\end{array}$ & 19 & $\begin{array}{l}\text { Ensino Fundamental } \\
\text { Incompleto }\end{array}$ & 18 \\
\hline
\end{tabular}

Fonte: Elaboração própria

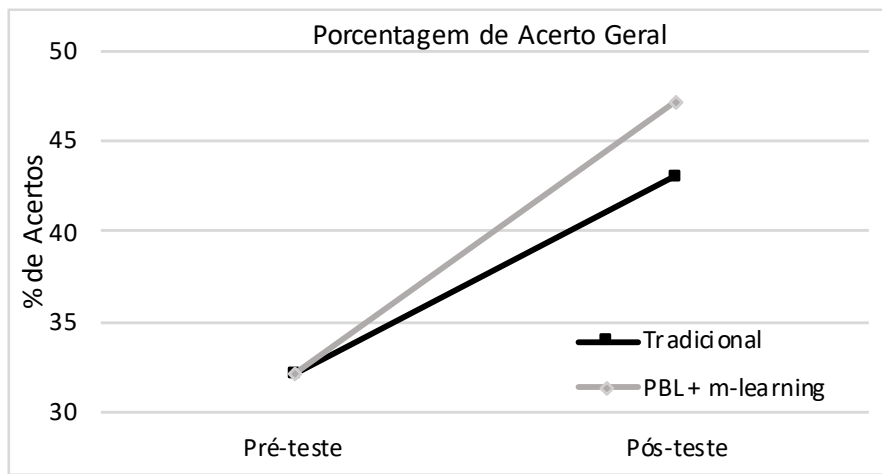

Figura 1 - Porcentagem de acerto geral dos métodos tradicional e PBL + $m$ learning na disciplina matemática.

A partir dos resultados apresentados na Figura 1, é possível verificar que os participantes submetidos ao método PBL+ m-learning alcançaram desempenho $8.68 \%$ superior aos alunos submetidos somente ao ensino tradicional. As análises seguintes irão apresentar somente os resultados de pré e pós-teste desse método. Assim esses resultados vão equiparar aos resultados previamente obtidos por Hmelo-Silver (2004) Dos Santos et. al. (2008).

A Figura 2 apresenta o desempenho dos alunos que usaram o método PBL $+m$ learning nas fases de pré e pós-teste. Neste resultado são apresentados os alunos com perfis sobre as condições de moradia e participação em programas sociais do governo. Desta forma, os resultados do pré e pós-teste são classificados em: (Perfil 1) alunos que moram em imóvel alugado e recebem auxilio de programas sociais; (Perfil 2) alunos que moram em casa própria e recebem auxílio de programas sociais; (Perfil 3) alunos tem imóvel próprio e não recebem auxílio de programas sociais. (Perfil 4) alunos que moram 
em imóvel alugados e não recebem benefícios de programas sociais. Para esse último caso, não houve presença de participantes com esse perfil no estudo.

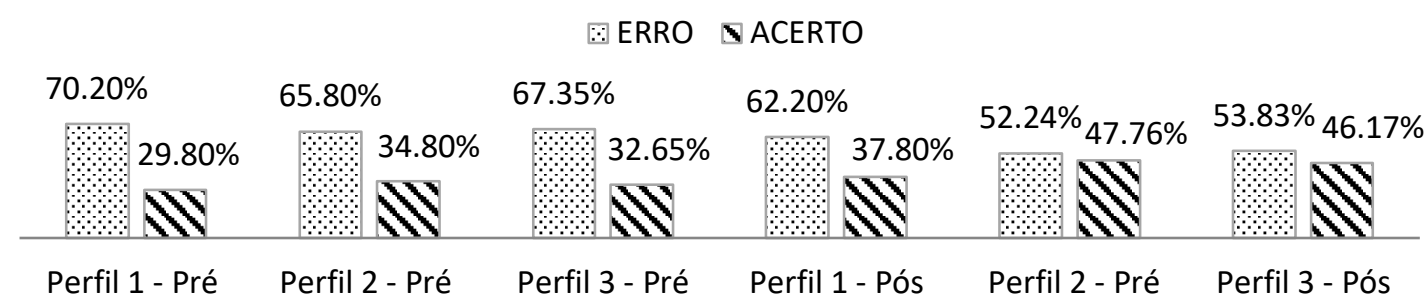

Figura 2 - Análise Tridimensional Considerando o Tipo de Moradia, Presença/Ausência de Programas Sociais, e Aplicação do método PBL + mlearning.

Para o Perfil 1, é possível observar que o nível de acerto no pré-teste foi de apenas $29,80 \%$, enquanto que durante o pós-teste foi obtido um percentual de acerto de $37,80 \%$, isto é um aumento de $8 \%$. Já para o Perfil 2, observa-se também que após a aplicação do método PBL+ m-learning, o rendimento do pós-teste obteve um aumento na precisão de desempenho em relação ao pré-teste. Sendo o percentual de acerto do préteste de $34,80 \%$, e no pós-teste de $47,76 \%$, aumento de $13 \%$. Para o Perfil 3, no pré-teste obteve-se um percentual de acerto igual a 32,65\%, e a partir da aplicação do método PBL+ m-learning, esse percentual saltou para 46,17\%, mostrando um aumento de $14 \%$.

A partir dos resultados apresentados na Figura 2, destacamos que os alunos com o Perfil 1 possuem o maior índice de erros na etapa pré-teste. Este resultado demostra que alunos que moram em imóvel alugado, isto é, com perfil semelhante a baixa renda, podem ser mais vulneráveis às falhas do ensino. Além disso, os resultados indicam que após a aplicação do método PBL+ m-learning, todos os indivíduos distribuídos nos três perfis obtiveram aumento na precisão de desempenho de modo independente aos fatores sociais e econômicos. Deste modo, indicamos que o método PBL+ $m$-learning sofre influência de fatores sociais e econômicos.

O Figura 3 apresenta o desempenho dos alunos durante os testes considerando os perfis que fazem referência às condições do bairro e acesso à Internet e ferramentas digitais. Os resultados do pré-teste e pós-teste são classificados em: (Perfil 1) alunos que moram na periferia e possuem acesso à Internet e ferramentas digitais; (Perfil 2) alunos que moram no centro e possuem acesso à Internet e ferramentas digitais; (Perfil 3) alunos que moram na periferia e não possuem acesso à Internet e ferramentas digitais; (Perfil 4) alunos que moram no centro e não possuem acesso à Internet e ferramentas digitais. Não houve presença de participantes com o Perfil 4.

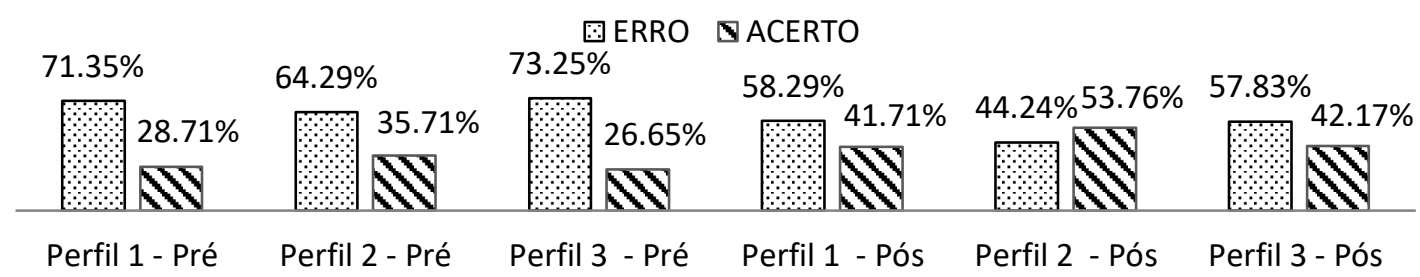

Figura 3 - Análise Tridimensional Considerando a Infraestrutura Social, a Infraestrutura Educacional, e Aplicação do método PBL + m-learning.

A partir da Figura 3, é possível observar que no Perfil 1 o nível de acerto no préteste foi de apenas $28,71 \%$, enquanto que foi obtido um percentual de acerto de 43,71 , isto é um aumento de $15 \%$. Já para o Perfil 2, destacamos o melhor índice de acertos entres todos os perfis avaliados. O percentual de acerto no pré-teste foi de $35,71 \%$, 
observa-se que após a aplicação do método PBL+ m-learning, o índice no pós-teste foi de $55,76 \%$; o que mostra um aumento de $20 \%$, sendo o único com resultado positivo entre todos os perfis apresentados. Quanto ao Perfil 3, comparado aos outros perfis, verificamos o menor índice de acertos na etapa pré-teste, sendo o percentual nessa fase apenas $26,65 \%$. A partir da aplicação da metodologia PBL+m-learning, obteve-se um percentual de desempenho de $42,17 \%$, mostrando um aumento de $16 \%$.

A partir dos resultados apresentados na Figura 3, destacamos que os alunos com Perfil 1 possuem maior índice de erros na etapa pré-teste. Este resultado aponta que alunos que moram na periferia e não possuem acesso à Internet podem vir a sofrer um maior impacto quando avaliados pelos métodos de ensino PBL+ m-learning. Enquanto que os alunos que moram no centro e possuem acesso à Internet (Perfil 2) tendem a ter um melhor desempenho quando avaliados pelo método PBL $+m$-learning. Tais resultados estatísticos estão em consonância com Freire (1994) que já assinalava que a diferença do ensino entre as classes sociais resultaria em diferentes resultados alcançados.

Por fim, os resultados também indicam que embora haja perda ocasionada por fatores sociais e econômicos, a aplicação do método PBL+ m-learning resulta positivamente no desempenho dos indivíduos distribuídos nos três perfis analisados. Deste modo, demonstrando que a aliança entre a tecnologia da informação com metodologias pedagógicas pode ser um caminho viável para encurtar as diferenças entre alunos advindos de diferentes contextos social e econômico.

\section{Conclusão}

O presente trabalho é o primeiro esforço em analisar o impacto da utilização conjunta das metodologias baseadas em PBL e em m-learning como estratégia de aprendizagem de matemática, assim como, é o primeiro que analisa de forma estatística o impacto de fatores sociais e econômicos em relação ao alcance pleno do ensino e aprendizagem.

Os resultados obtidos demonstram que a metodologia PBL + m-learning se destaca em relação ao método de ensino tradicional. Além disso, os resultados indicam que alunos com melhor infraestrutura educacional tendem a obter melhores desempenhos usando a metodologia PBL $+m$-learning. Também é possível observar a partir dos resultados que mesmo os alunos que não dispõem de uma melhor infraestrutura educacional também podem obter melhoras no desempenho usando o método PBL $+m$ learning quando comparado aos alunos que foram submetidos ao método tradicional.

Em consonância com Freire (1994) que já assinalava em seu texto a diferença do ensino entre as classes sociais. A partir deste trabalho é possível concluir que diferentes métodos de ensino, por exemplo, tradicional ou PBL $+m$-learning, sofrem influências de fatores sociais e econômicos. Este trabalho é o primeiro esforço que objetiva apresentar de forma estatística a diferença no desempenho dos alunos oriundos de situações de vulnerabilidade socioeconômica em relação a alunos que vem de melhores condições de ensino e melhor infraestrutura educacional. Desta forma, os resultados obtidos ratificam o conceito de que o histórico social e econômico de determinado aluno oriundo da periferia ou que necessidade de auxilio de programas sociais para garantir suas condições de existência digna, podem causar diferença negativa, quando comparado ao de um aluno oriundo de condições sociais e econômica mais favoráveis.

Desta forma, este trabalho evidencia de forma estatística o valor do impacto causado, assim demonstrando a necessidade evidente da implantação de políticas que permitam o maior acesso à igualdade de direitos entre os indivíduos. Já que "a educação é direito de todos e dever do estado" (Brasil Senado Federal, 1988). 
Os resultados deste trabalho estão de acordo com o pensamento de Leite (2005), onde é destacado o papel do PBL como ferramenta de suporte ao processo de ensinoaprendizagem. Além disso, os resultados obtidos demonstram que o uso do acesso a diferentes recursos tecnológicos pode ser um caminho para a melhoria do desempenho dos alunos, melhoria da motivação da participação dos alunos e um caminho para a redução da diferença de aprendizado causada por fatores advindos da biografia dos estudantes que são associados aos seus históricos sociais e econômicos.

Além disso, o os resultados obtidos provam estatisticamente que a metodologia $\mathrm{PBL}+m$-learning pode ser uma alternativa eficiente para reduzir a diferença, pois ambos os perfis avaliados no experimento tiveram melhoria no desempenho após a aplicação do método. A partir disso e da análise tridimensional (PBL + m-learning, social e econômica), podemos concluir que o $\mathrm{PBL}+$ m-learning podem ajudar a complementar o processo de ensino de aprendizagem ativo a ser adotado dentro da escola de forma democrática, isto é, sofrendo baixa influência de fatores sociais e econômicos.

Para trabalhos futuros, espera-se expandir o número de alunos participantes do experimento, assim como, aplicar a metodologia em diferentes contextos socioeconômicos e em escolas localizadas em diferentes bairros da região.

\section{Referências Bibliográficas}

ANDRADE, M. A. B. S. et al. Possibilidades e limites da prática da aprendizagem baseada em problemas (PBL) no ensino médio. Enseñanza de las Ciencias, n. Extra, p. $1-3,2005$.

BATISTA, S. C. F.; BARCELOS, G. T. Análise do uso do celular no contexto educacional. RENOTE, v. 11, n. 1, 2017.

BRASIL SENADO FEDERAL. Constituição da república federativa do Brasil. Brasília: Senado Federal, Centro Gráfico, 1988.

DE OLIVEIRA, L. R.; MEDINA, R. D. Desenvolvimento de objetos de aprendizagem para dispositivos móveis: uma nova abordagem que contribui para a educação. RENOTE, v. 5, n. 1, 2007.

DOS SANTOS, S. C. et al. Usando pbl na qualificação de profissionais em engenharia de software. Anais do FEES08-Fórum de Educação em Engenharia de Software, 2008.

CYRINO, E. G.; TORALLES-PEREIRA, M. L. Trabalhando com estratégias de ensinoaprendizado por descoberta na área da saúde: a problematização e a aprendizagem baseada em problemas. Cadernos de Saúde Pública, v. 20, p. 780-788, 2004.

FADESP, Fundação Amazônia de Amparo a Estudos e Pesquisas. Barômetro da Sustentabilidade do Município de Bragança: Belém, 2016.

FELÍCIO, F.; FERNANDES, R. O efeito da qualidade da escola sobre o desempenho escolar: uma avaliação do ensino fundamental no estado de São Paulo. Anais do XXXIII Encontro Nacional de Economia, 2005.

FRANCISCATO, F. T.; MEDINA, R. D. M-Learning e Android: um novo paradigma?. RENOTE, v. 6, n. 1, 2008. 
FREIRE, P. Educação como prática da liberdade. Rio de Janeiro: Paz e Terra, 1994.

HMELO-SILVER, C. E. Problem-based learning: What and how do students learn?. Educational psychology review, v. 16, n. 3, p. 235-266, 2004.

JACOMINI, M. A. Educar sem reprovar: desafio de uma escola para todos. Educação e pesquisa, v. 35, n. 3, p. 557-572, 2009.

JUNG, H. J. Fostering an English Teaching Environment: factors influencing english as a foreign language teachers' adoption of mobile learning. Informatics in Education, DOI: 10.15388. Vol. 14, nº. 2, 219-241, 2015.

LAKATOS, E. M; MARCONI, M. A. Fundamentos de Metodologia Científica. $5^{\text {a }}$ ed. São Paulo: Atlas S.A. 2003.

LEITE, L; ESTEVES, E. Ensino orientado para a aprendizagem baseada na resolução de problemas na Licenciatura em Ensino de Física e Química. In SILVA, Bento D.; ALMEIDA, Leandro S., coord. - "Actas do Congresso Galaico-Português de Psicopedagogia, 8, Braga, Portugal, 2005” [CD-ROM]. Braga : Centro de Investigação em Educação do Instituto de Educação e Psicologia da Universidade do Minho, 2005. ISBN 972-8746-36-9. p. 1752-1768.

MEZZARI, A. O uso da Aprendizagem Baseada em Problemas (ABP) como reforço ao ensino presencial utilizando o ambiente de aprendizagem Moodle. Revista brasileira de educação médica. Vol. 35, n. 1 (2011), p. 114-121, 2011.

MIZUKAMI, M. G. N. Ensino: as abordagens do processo. São Paulo: EPU, 1986.

MOCBEL, M; FARIAS, F. Aplicativo Exatas: Ferramenta de apoio ao aprendizado móvel. In: Anais dos Workshops do Congresso Brasileiro de Informática na Educação. 2018. p. 172.

QUEIROZ, A. PBL, Problemas que trazem soluções. Revista Psicologia, Diversidade e Saúde, v. 1, n. 1, 2012.

RELATÓRIO ENEM 2018. Disponível em: <http://download.inep.gov.br/educacao_basica/enem/downloads/2018/presskit/presskit_ enem-resultados2018.pdf >. Acesso em: 18 de maio 2019.

RIBEIRO, L. R. C. Aprendizagem baseada em problemas (PBL) na educação em engenharia. Revista de Ensino de Engenharia, v. 27, n. 2, p. 23-32, 2008.

SALES, L. F. Aprendizagem Baseada em Problemas (PBL) no Curso de Medicina do Interior da Amazônia: uma análise do processo tutorial. Santarém. UFOPA, 2016.

TOP 10 GOOGLE PLAY CATEGORIES. Ranking de Categorias de Aplicativos. Disponível em: <https://www.appbrain.com/stats/android-market-app-categories $>$. Acesso em: 18 maio 2019. 